Supporting information for the manuscript entitled:

\title{
Highly Efficient Endoglycosidase-Catalyzed Synthesis of Glycopeptides Using Oligosaccharide Oxazolines as Donor Substrates
}

\author{
Bing Li, Ying Zeng, Steven Hauser, Haijing Song, and Lai-Xi Wang* \\ Institute of Human Virology, University of Maryland Biotechnology Institute, University of \\ Maryland, 725 West Lombard Street, Baltimore, MD 21201, USA \\ E-mail: wangx@umbi.umd.edu
}




\section{Materials and methods}

All chemicals, biochemicals and reagents were purchased from Sigma/Aldrich Chemical Company (St. Louis, MO). HPLC grade acetonitrile was purchased from Fisher Scientific. DMF was purchased from B \& J Biosynthesis. TLC was performed on glass plates coated with silica gel 60 F254 (E. Merck). Flash column chromatography was performed on silica gel 60 (EM Science, $230-400$ mesh). ${ }^{1} \mathrm{H}$ and ${ }^{13} \mathrm{C}$ NMR spectra were recorded on Inova 500 NMR machine. The ESI-MS spectra were measured on a micromass ZQ-4000 single quadruple mass spectrometer.

\section{Reverse-phase HPLC}

Analytical HPLC was carried out with a Waters 626 HPLC instrument on a Waters NovaPak C18 column $(3.9 \times 150 \mathrm{~mm})$ at $40{ }^{\circ} \mathrm{C}$. The column was eluted with a linear gradient of $0-90 \%$ $\mathrm{MeCN}$ containing $0.1 \%$ TFA at a flow rate of $1 \mathrm{~mL} / \mathrm{min}$ over 25 minutes. Peptides and glycopeptides were detected at double wavelengths (214 and $280 \mathrm{~nm})$. Preparative HPLC was performed with a Waters 600 HPLC instrument of a Waters C18 column (Symmetry 300, $19 \times 300 \mathrm{~mm}$ ). The column was eluted with a suitable gradient of MeCN containing $0.1 \% \mathrm{TFA}$ at $12 \mathrm{~mL} / \mathrm{min}$.

\section{Dionex high-performance anion exchange chromatography coupled with pulsed electrochemical detection (HPAEC-PED)}

The analytical anion-exchange chromatography was performed on a Dionex DX600 chromatography system (Dionex Corporation, Sunnyvale, CA) equipped with an electrochemical detector (ED50, Dionex Corporation, Sunnyvale, Calif.). The following conditions were used: column, CarboPac-PA1 (4x250mm); Eluent A, 0.1 M NaOH; Eluent B, 1 M sodium acetate (NaOAc) in $0.1 \mathrm{M} \mathrm{NaOH}$; Gradient: 0-5 min, 0\% B; $5-25 \mathrm{~min}, 0-15 \% \mathrm{~B}$. Flow rate, $1 \mathrm{ml} / \mathrm{min}$.

\section{Peptide synthesis}

The GlcNAc-peptides were synthesized according to the reported procedures ( $\mathrm{Li}, \mathrm{H}$. et al., Bioorg Med Chem Lett, 2005, 15, 895; Wang, L. X. et al, ChemBioChem, 2005, in press). Briefly, Peptides were assembled on a on a Pioneer automatic peptide synthesizer (Applied Biosystems) using fluorenylmethyloxycarbonyl (Fmoc)-protected amino acids and FmocAsn(Ac3GlcNAc)-OH as the building blocks, 2-(1-H-azabenzotriazol-1-yl)-1,1,3,3tetramethyluronium hexafluorophosphate (HATU) as the coupling reagent, and polyethylene glycol-polystyrene resin with a peptide amide linker (PAL-PEG-PS resin) as the solid support. The peptides were retrieved from the resin with simultaneous deprotection by treatment with cocktail R (TFA/thioanisole/EDT/anisole, 90:5:3:2) and precipitated by cold ether. The crude peptides were subject to the treatment with 5\% aqueous hydrazine to remove the O-acetyl groups, and the resulting crude GlcNAc-peptides were purified by RP-HPLC to provide the GlcNAc-heptapeptide (12) and the 34-mer peptide GlcNAc-C34.

\section{Synthesis of the oligosaccharide oxazolines}


Benzyl O-(2-O-acetyl-4,6-O-benzylidene-3-O-p-methoxybenzyl- $\beta$-D-glucopyranosyl) -(1 $\rightarrow 4)-3,6-$ di-O-benzyl-2-deoxy-2-phthalamido- $\beta$-D-glucopyranoside $(3)$.

A suspension of compound 1 (340 mg, $0.72 \mathrm{mmol})$ and compound 2 (390 $\mathrm{mg}, 0.68$ mmol) containing activated $4 \AA$ molecular sieves $(200 \mathrm{mg})$ in dry $\mathrm{CH}_{2} \mathrm{Cl}_{2}(10 \mathrm{ml})$ was stirred under an atmosphere of argon at r.t. for $30 \mathrm{~min}$. After cooling to $-40^{\circ} \mathrm{C}$, NIS $(170 \mathrm{mg}, 0.76$ mmol) and AgOTf $(18 \mathrm{mg}, 0.07 \mathrm{mmol})$ were added and the mixture was stirred at $-40^{\circ} \mathrm{C}$ to r.t. overnight. The reaction was quenched with $\mathrm{Et}_{3} \mathrm{~N}$, washed with brine, dried over $\mathrm{Na}_{2} \mathrm{SO}_{4}$, and filtered. The filtrate was concentrated in vacuo and the residue was subjected to flash silica gel column chromatography (6:1 Hexanes: EtOAc) to afford $3(490 \mathrm{mg}, 74 \%)$ as a white foam. ${ }^{1} \mathrm{H}$ NMR $\left(\mathrm{CDCl}_{3}, 500 \mathrm{MHz}\right): \delta$ 7.71-6.90 (m, 28H, Ar), $5.52\left(\mathrm{~s}, 1 \mathrm{H}, \mathrm{PhC}_{\mathrm{HO}}{ }^{-}\right), 5.14(\mathrm{~d}, 1 \mathrm{H}$, $\mathrm{J}=7.5 \mathrm{~Hz}, \mathrm{H}-1), 5.01$ (t, 1H, J=8.5Hz, H-2'), 4.86-4.82 (m, 3H), $4.78\left(\mathrm{~d}, 1 \mathrm{H}, \mathrm{J}=12.5 \mathrm{~Hz}, \mathrm{PhCH}_{2}{ }^{-}\right)$, 4.65 (s, 1H, H-1'), 4.63 (d, J=12Hz, $\left.\mathrm{PhC}_{2}{ }_{2}\right), 4.54$ (d, J=12Hz, 1H, $\mathrm{PhC}_{\mathrm{H}_{2}}$ ), 4.52 (d, J=12.5 $\mathrm{Hz}$, $\left.1 \mathrm{H}, \mathrm{PhCH}_{2}-\right), 4.45\left(\mathrm{~d}, \mathrm{~J}=12.5 \mathrm{~Hz}, 1 \mathrm{H}, \mathrm{PhCH}_{2}-\right), 4.30$ (dd, J=4.5Hz, $10.5 \mathrm{~Hz}, 1 \mathrm{H}, \mathrm{H}-6$ ') $), 2.26$ (t, $\mathrm{J}=4 \mathrm{~Hz}, 1 \mathrm{H}, \mathrm{H}-2), 4.12(\mathrm{t}, \mathrm{J}=9.5 \mathrm{~Hz}, 1 \mathrm{H}, \mathrm{H}-3), 3.85-3.81(\mathrm{~m}, 5 \mathrm{H}), 3.69$ (t, J=9.5Hz, 1H, H-4'), 3.60 (t, J=9.0Hz, 1H, H-3'), 3.56-3.50(m, 3H), 3.29-3.26(m, 1H, H-5'), 2.04(s, 3H, $\left.\mathrm{C}_{3} \mathrm{CO}_{2^{-}}\right)$; ${ }^{13} \mathrm{C} \mathrm{NMR}\left(\mathrm{CDCl}_{3}, 125 \mathrm{MHz}\right): \delta 169.1,138.5,138.0,137.3,137.2,130.4,129.4,128.6,128.3$, 128.2, 128.1, 127.9, 127.8, 127.6, 127.1, 126.1, 101.2, 100.8, 97.4, 81.8, 78.0, 76.6, 74.9, 74.6, $73.7,73.4,70.8,68.7,67.6,66.0,55.7,55.3$.

Benzyl O-(2-O-benzoyl-4,6-O-benzylidene-3-O-p-methoxybenzyl- $\beta$-D-mannopyranosyl)-(1 $\rightarrow$ 4)-3,6-di-O-benzyl-2-deoxy-2-phthalamido- $\beta$-D-glucopyranoside (4).

A solution of compound 3 (170 mg, $0.18 \mathrm{mmol})$ in $\mathrm{MeOH}(2 \mathrm{ml})$ containing $\mathrm{NaOMe}(2$ $\mathrm{mg}, 40 \mu \mathrm{mol})$ was stirred at r.t. for $8 \mathrm{~h}$. The reaction was neutralized by Dowex W50-X8 $\left(\mathrm{H}^{+}\right.$ form) and concentrated in vacuo. The foregoing compound ( $160 \mathrm{mg}, 0.18 \mathrm{mmol})$ in $\mathrm{CH}_{2} \mathrm{Cl}_{2}(3$ $\mathrm{ml})$ was treated with Py $(290 \mu 1,3.59 \mathrm{mmol})$ and $\mathrm{Tf}_{2} \mathrm{O}(240 \mu 1,1.43 \mathrm{mmol})$ at $0^{\circ} \mathrm{C}$. The reaction was monitored by TLC. After the completion of the reaction, the mixture was diluted with $\mathrm{CH}_{2} \mathrm{Cl}_{2}$, washed successively with cold $\mathrm{HCl}(0.1 \mathrm{~N})$, cold saturated $\mathrm{NaHCO}_{3}$ and $\mathrm{H}_{2} \mathrm{O}$, and dried over $\mathrm{Na}_{2} \mathrm{SO}_{4}$. The filtrate was concentrated and coevaporated with toluene. The residue was dissolved in toluene $(10 \mathrm{ml})$ and $\mathrm{Bu}_{4} \mathrm{NOBz}(500 \mathrm{mg}, 1.38 \mathrm{mmol})$ was added. The mixture was stirred under reflux for $2 \mathrm{~h}$ when TLC indicated the completion of the reaction. After evaporation, the mixture was diluted with $\mathrm{CH}_{2} \mathrm{Cl}_{2}$, washed successively with saturated $\mathrm{NaHCO}_{3}$ and $\mathrm{H}_{2} \mathrm{O}$, dried over $\mathrm{Na}_{2} \mathrm{SO}_{4}$, and filtered. The filtrate was concentrated in vacuo and the residue was subjected to flash silica gel column chromatography (5:1 Hexanes: EtOAc) to afford 4 (140 mg, $81 \%)$ as a white foam. ${ }^{1} \mathrm{H} \mathrm{NMR}\left(\mathrm{CDCl}_{3}, 500 \mathrm{MHz}\right): \delta 8.17-6.75(\mathrm{~m}, 33 \mathrm{H}, \mathrm{Ar}), 5.75(\mathrm{~d}, 1 \mathrm{H}$, $\left.\mathrm{J}=3.5 \mathrm{~Hz}, \mathrm{H}-2^{\prime}\right), 5.63\left(\mathrm{~s}, 1 \mathrm{H}, \mathrm{PhCHO}_{2^{-}}\right), 5.10(\mathrm{~d}, \mathrm{~J}=8.0 \mathrm{~Hz}, 1 \mathrm{H}, \mathrm{H}-1), 4.86(\mathrm{~d}, \mathrm{~J}=12 \mathrm{~Hz}, 1 \mathrm{H}$,

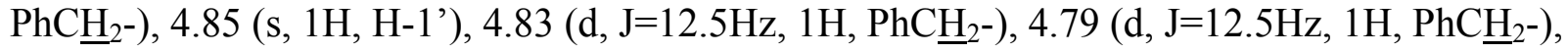

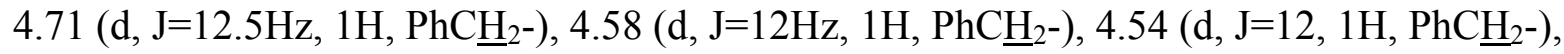

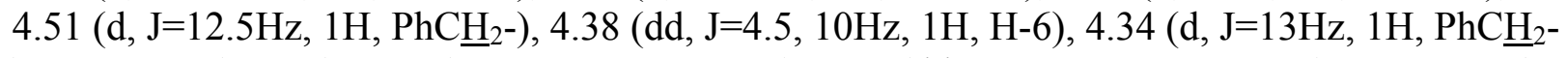
), 4.24-4.10 (m, 5H), 4.04 (t, J=9.5Hz, 1H, H-4'), 3.93 (dd, J=3, 10Hz, 1H, H-6' ), 3.86-3.77 (m, $6 \mathrm{H}), 3.64-3.58$ (m, 2H), 3.30-3.29 (m, H-5); $\left.{ }^{13} \mathrm{C} \mathrm{NMR} \mathrm{CDCl}_{3}, 125 \mathrm{MHz}\right)$ : 165.8, 138.5, 137.9, 137.6, 137.2, 133.2, 130.0, 129.3, 129.0, 128.7, 128.5, 128.2, 128.1, 128.0, 127.7, 127.6, 127.0, 126.2 , 101.6, 99.5, 97.4, 79.1, 78.4, 76.4, 75.6, 74.5, 74.4, 73.7, 71.3, 70.8, 69.7, 68.8, 68.5, 67.1, $55.7,55.3$.

\footnotetext{
Benzyl O-(2-O-benzoyl-4,6-O-benzylidene- $\beta$-D-mannopyranosyl)-(1 $\rightarrow 4)-3,6-$ di-Obenzyl -2-deoxy-2-phthalamido- $\beta$-D-glucopyranoside (5).
} 
To a stirring solution of compound 4 (440 $\mathrm{mg}, 0.45 \mathrm{mmol})$ in $\mathrm{CH}_{2} \mathrm{Cl}_{2}(5 \mathrm{ml})$ and $\mathrm{H}_{2} \mathrm{O}$ $(0.5 \mathrm{ml})$ was added DDQ $(120 \mathrm{mg}, 0.53 \mathrm{mmol})$. After stirring for $9 \mathrm{~h}$, the reaction mixture was diluted with $\mathrm{CH}_{2} \mathrm{Cl}_{2}$. The organic layer was washed with saturated $\mathrm{NaHCO}_{3}$ and $\mathrm{H}_{2} \mathrm{O}$, dried by $\mathrm{Na}_{2} \mathrm{SO}_{4}$, and filtered. The filtrate was concentrated in vacuo and the residue was subjected to flash silica gel column chromatography (3:1 Hexanes: EtOAc) to give $5(290 \mathrm{mg}, 75 \%)$ as a white foam. ${ }^{1} \mathrm{H} \mathrm{NMR}\left(\mathrm{CDCl}_{3}, 500 \mathrm{MHz}\right): \delta 8.17-6.75$ (m, 29H, Ar), 5.59 (s, 2H, H-2', $\mathrm{PhCHO}_{2-}$ ), 5.09 (d, J=8.5Hz, 1H, H-1), 4.90 (s, 1H, H-1'), 4.89 (d, J=12.5Hz, 1H, $\mathrm{PhC}_{2}-$ ), 4.82 (d, J=12, $\left.1 \mathrm{H}, \mathrm{PhC}_{\mathrm{H}_{2}}\right), 4.78\left(\mathrm{~d}, \mathrm{~J}=12 \mathrm{~Hz}, 1 \mathrm{H}, \mathrm{PhCH}_{2}-\right), 4.58\left(\mathrm{~d}, \mathrm{~J}=11.5 \mathrm{~Hz}, 1 \mathrm{H}, \mathrm{PhCH}_{2}-\right), 4.51(\mathrm{~d}$, $\left.\mathrm{J}=12.5 \mathrm{~Hz}, 1 \mathrm{H}, \mathrm{PhCH}_{2}-\right), 4.39-4.32(\mathrm{~m}, 2 \mathrm{H}), 4.24-4.09(\mathrm{~m}, 4 \mathrm{H}), 3.96-3.73(\mathrm{~m}, 6 \mathrm{H}), 3.56(\mathrm{~d}, \mathrm{~J}=9$, 1H), 3.32-3.27 (m, 1H, H-5); ${ }^{13} \mathrm{C} \mathrm{NMR}\left(\mathrm{CDCl}_{3}, 125 \mathrm{MHz}\right): \delta 166.03,138.4,137.97,137.19$, 133.53, 133.30, 130.0, 128.7, 128.5, 128.4, 128.2, 128.2, 128.1, 127.9, 127.7, 127.6, 127.0, 126.3, 102.2, 99.5, 97.4, 79.4, 79.1, 76.4, 74.5, 74.4, 73.7, 71.9, 70.8, 70.0, 68.7, 68.4, 66.8, 55.7 .

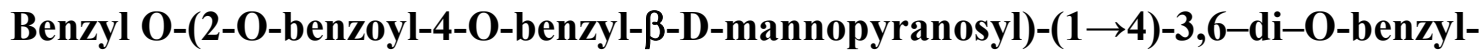 2-deoxy-2-phthalamido- $\beta$-D-glucopyranoside (6).}

To a solution of compound $\mathbf{5}(150 \mathrm{mg}, 0.160 \mathrm{mmol})$ in dry THF $(1 \mathrm{ml})$ containing $\mathrm{BH}_{3}(1$ mmol) was added a solution of $\mathrm{Bu}_{2} \mathrm{BOTf}$ in $\mathrm{CH}_{2} \mathrm{Cl}_{2}(1 \mathrm{M}, 180 \mu \mathrm{l})$ at $0^{\circ} \mathrm{C}$ and the mixture was stirred at $0^{\circ} \mathrm{C}$ for $3 \mathrm{~h}$ when TLC indicated the completion of the reaction. $\mathrm{Et}_{3} \mathrm{~N}$ was added to the reaction followed by careful addition of $\mathrm{MeOH}$ till the evolution of $\mathrm{H}_{2}$ had ceased. The mixture was coevaporated with $\mathrm{MeOH}$ three times and the residue was subjected to flash silica gel column chromatography (2:1 Hexanes: EtOAc) to afford 6 ( $120 \mathrm{mg}, 78 \%$ ) as a white foam. ${ }^{1} \mathrm{H}$ NMR ( $\left.\mathrm{CDCl}_{3}, 500 \mathrm{MHz}\right): \delta 8.13-6.77$ (m, 29H, Ar), 5.51 (s, 1H, H-2'), 5.11 (d, J=8Hz, 1H, H1), 4.88-4.82 (m, $5 \mathrm{H}), 4.71$ (d, J=11.5Hz, $\left.1 \mathrm{H}, \mathrm{PhC}_{2} 2^{-}\right), 4.58$ (d, J=12.5Hz, $\left.1 \mathrm{H}, \mathrm{PhCH}_{2}-\right), 4.52$ (d, $\left.\mathrm{J}=12,1 \mathrm{H}, \mathrm{PhC}_{2}-\right), 4.35$ (d, J=12, 1H, $\left.\mathrm{PhCH}_{2}-\right), 4.25(\mathrm{t}, \mathrm{J}=9.5 \mathrm{~Hz}, 1 \mathrm{H}, \mathrm{H}-4), 4.19-4.13(\mathrm{~m}, 3 \mathrm{H})$, 3.92-3.59 (m, 9H), 3.33-3.31 (m, $1 \mathrm{H}, \mathrm{H}-5) ;{ }^{13} \mathrm{C} \mathrm{NMR}\left(\mathrm{CDCl}_{3}, 125 \mathrm{MHz}\right): \delta 166.2,138.3,138.1$, $137.9,137.2,133.6,133.3,131.6,130.0,128.7,128.6,128.5,128.2,128.1,128.0,127.7,127.6$, 127.4, 127.1, 98.6, 97.4, 78.8, 76.5, 75.6, 74.9, 74.5, 74.3, 73.7, 73.2, 72.3, 70.8, 68.4, 62.1, 55.7.

Benzyl O-[(2,3,4,6-tetra-O-acetyl- $\alpha$-D-mannopyranosyl)-(1 $\rightarrow$ 6)-(2,3,4,6-tetra-Oacetyl - $\alpha$-D-mannopyranosyl)-(1 $\rightarrow 3$ )-2-O-benzoyl-4-O-benzyl- $\beta$-D-mannopyranosyl]$(1 \rightarrow 4)-3,6-d i-O-b e n z y l-2-d e o x y-2-p h t h a l a m i d o-\beta$-D-glucopyranoside (7).

A suspension of compound $\mathbf{6}(220 \mathrm{mg}, 0.24 \mathrm{mmol})$ and 2,3,4,6-tetra-O-acetyl- $\alpha$-Dmannopyranosyl tricholoroacetimidate $(590 \mathrm{mg}, 1.20 \mathrm{mmol})$ in dry $\mathrm{CH}_{2} \mathrm{Cl}_{2}(4 \mathrm{ml})$ containing activated $4 \AA$ molecular sieves $(100 \mathrm{mg})$ was stirred under an atmosphere of argon at r.t. for 30 min. After cooling to $-78{ }^{\circ} \mathrm{C}$, a solution of TMSOTf in $\mathrm{CH}_{2} \mathrm{Cl}_{2}(0.1 \mathrm{M}, 200 \mu \mathrm{l}, 20 \mu \mathrm{mol})$ was added and the resulting mixture was stirred at r.t. for $5 \mathrm{~h}$. The reaction was quenched with $\mathrm{Et}_{3} \mathrm{~N}$, washed with brine, dried over $\mathrm{Na}_{2} \mathrm{SO}_{4}$, and filtered. The filtrate was concentrated in vacuo and the residue was subjected to flash silica gel column chromatography (6:1 Hexanes: EtOAc) to provide $7(300 \mathrm{mg}, 80 \%)$ as a white foam. ${ }^{1} \mathrm{H} \mathrm{NMR}\left(\mathrm{CDCl}_{3}, 500 \mathrm{MHz}\right): \delta 8.12-6.49(\mathrm{~m}, 29 \mathrm{H}$, Ar), $5.64(\mathrm{~d}, \mathrm{~J}=3.5 \mathrm{~Hz}, 1 \mathrm{H}, \mathrm{H}-1), 5.46-5.30(\mathrm{~m}, 5 \mathrm{H}), 5.21(\mathrm{dd}, \mathrm{J}=3.5,10.5 \mathrm{~Hz}, 1 \mathrm{H}), 5.14(\mathrm{~s}, 1 \mathrm{H}, \mathrm{H}-$ 1'”), $5.03(\mathrm{~d}, \mathrm{~J}=8.5 \mathrm{~Hz}, 1 \mathrm{H}), 4.93-4.90(\mathrm{~m}, 3 \mathrm{H}), 4.78\left(\mathrm{~d}, \mathrm{~J}=10 \mathrm{~Hz}, 1 \mathrm{H}, \mathrm{PhCH}_{2}-\right), 4.77$ (s, 1H, H1'), 4.67 (d, J=12Hz, $\left.1 \mathrm{H}, \mathrm{PhCH}_{2}-\right), 4.58$ (d, J=13.5Hz, $\left.1 \mathrm{H}, \mathrm{PhCH}_{2}-\right), 4.53(\mathrm{~d}, \mathrm{~J}=12 \mathrm{~Hz}, 1 \mathrm{H}$, $\mathrm{PhC}_{2_{2}}$ ), $4.45\left(\mathrm{~d}, \mathrm{~J}=12 \mathrm{~Hz}, 1 \mathrm{H}, \mathrm{PhC}_{\mathrm{H}_{2}}\right), 4.38(\mathrm{dd}, \mathrm{J}=3.0,12.5 \mathrm{~Hz}, 1 \mathrm{H}), 4.28(\mathrm{~d}, \mathrm{~J}=4,12.5 \mathrm{~Hz}$, $1 \mathrm{H}), 4.24-4.18(\mathrm{~m}, 4 \mathrm{H}), 4.12-3.98(\mathrm{~m}, 4 \mathrm{H}), 3.92-3.81(\mathrm{~m}, 5 \mathrm{H}), 3.76(\mathrm{dd}, \mathrm{J}=3.0,9.5 \mathrm{~Hz}, 1 \mathrm{H}), 3.53$ (d, J=9.5Hz, 1H), 3.35-3.32 (m, 1H, H-5), 2.19, 2.14, 2.13, 2.12, 2.07, 2.00, 1.98, 1.96 (s each, $3 \mathrm{H}$ each, $\left.8 \mathrm{xC}_{3} \mathrm{CO}_{2}-\right) ;{ }^{13} \mathrm{C} \mathrm{NMR}\left(\mathrm{CDCl}_{3}, 125 \mathrm{MHz}\right): \delta 170.7,170.2,169.9,169.8,169.7$, 
$169.5,167.3,165.8,138.1,138.0,137.7,137.2,133.4,130.1,129.4,128.8,128.7,128.5,128.4$, $128.3,128.1,127.9,127.6,127.5,126.8,99.9,98.0,97.8,97.4,80.4,78.0,75.9,75.3,74.9,74.5$, $74.4,73.7,71.3,70.8,69.6,69.5,69.4,69.3,68.9,68.7,68.1,66.7,65.8,65.6,62.5,62.3,55.7$.

O-[(2,3,4,6-tetra-O-acetyl- $\alpha$-D-mannopyranosyl)- $(1 \rightarrow 6)-(2,3,4,6$-tetra-O-acetyl- $\alpha-D-$ mannopyranosyl)-(1 $\rightarrow 3)-2,4-d i-O$-acetyl- $\beta$-D-mannopyranosyl]-(1 $\rightarrow 4)$-2-acetamido-1,3,6tri-O-acetyl-2-deoxy- $\alpha$ - and $\beta$-D-glucopyranose (8).

A mixture of $7(71 \mathrm{mg}, 40 \mu \mathrm{mol})$ and $\mathrm{NH}_{2} \mathrm{NH}_{2} \cdot \mathrm{H}_{2} \mathrm{O}(3 \mathrm{ml})$ in EtOH $(27 \mathrm{ml})$ was heated at $90^{\circ} \mathrm{C}$ for $24 \mathrm{~h}$ and then concentrated in vacuo. To the oily residue were added pyridine $(2 \mathrm{ml})$ and $\mathrm{Ac}_{2} \mathrm{O}(2 \mathrm{ml})$. The mixture was stirred at r.t. overnight. The mixture was diluted with $\mathrm{CH}_{2} \mathrm{Cl}_{2}(20$ ml) and washed with saturated $\mathrm{NaHCO}_{3}, \mathrm{HCl}(1 \mathrm{~N})$ and $\mathrm{H}_{2} \mathrm{O}$, dried over $\mathrm{Na}_{2} \mathrm{SO}_{4}$, and filtered. The filtrate was concentrated in vacuo and the residue was subject to flash silica gel column chromatography (1:3 Hexanes: EtOAc) to give the corresponding 2-deoxy-2-acetamido compound (46 mg, 78\%) as a white foam. ${ }^{1} \mathrm{H} \mathrm{NMR}\left(\mathrm{CDCl}_{3}, 500 \mathrm{MHz}\right): \delta 7.42-7.30(\mathrm{~m}, 20 \mathrm{H}$, Ar), 5.84 (d, J=8Hz, 1H, H-1), 5.39-5.25 (m, 6H), 5.26 (dd, J=3, 10Hz, 1H), 5.14 (s, 1H, H-1 ', '), 4.91-4.87 (m, $4 \mathrm{H}), 4.84$ (d, J=12.5Hz, $\left.1 \mathrm{H}, \mathrm{PhCH}_{2}-\right), 4.78$ (d, J=12Hz, 1H, $\left.\mathrm{PhCH}_{2}{ }^{-}\right), 4.68(\mathrm{~s}, 1 \mathrm{H}$, H-1'), 4.65 (d, J=11.5Hz, 1H, $\left.\mathrm{PhCH}_{2}-\right), 4.61$ (d, J=11Hz, $\left.1 \mathrm{H}, \mathrm{PhCH}_{2}-\right), 4.58(\mathrm{~d}, \mathrm{~J}=12,1 \mathrm{H}$, $\left.\mathrm{PhCH}_{2}-\right)$, 4.51 (d, J=12, 1H, $\mathrm{PhCH}_{2}-$ ), 4.39-4.33 (m, 2H), 4.20-4.17 (m, 3H), 4.08-4.02 (m, 2H), $3.96(\mathrm{t}, \mathrm{J}=9 \mathrm{~Hz}, 1 \mathrm{H}), 3.80(\mathrm{~d}, \mathrm{~J}=3.5 \mathrm{~Hz}, 1 \mathrm{H}), 3.75-3.69(\mathrm{~m}, 4 \mathrm{H}), 3.62-3.51(\mathrm{~m}, 3 \mathrm{H}), 3.28-3.25(\mathrm{~m}$, $1 \mathrm{H}), 2.19,2.15,2.13,2.12,2.09,2.08,2.04,2.03,1.98,1.89$ (s each, $3 \mathrm{H}$ each, $\left.10 \mathrm{xC}_{\underline{3}} \mathrm{CO}_{2^{-}}\right) ;{ }^{13} \mathrm{C}$ $\mathrm{NMR}\left(\mathrm{CDCl}_{3}, 125 \mathrm{MHz}\right): \delta 171.0,170.6,170.4,169.9,169.8,169.7,167.8,138.6,138.1,137.6$, $137.4,132.5,130.9,128.8,128.6,128.5,128.3$, 128.2 , 128.0, 127.9, 127.8, 127.7, 127.6, 99.6, $99.5,97.5,97.0,78.3,77.7,76.1,75.2,74.8,74.5,73.9,73.5,70.9,70.8,69.5,69.4,68.9,68.6$, $68.4,68.2,66.4,65.7,65.5,62.4,55.8$.

A mixture of the 2-acetamido-2-deoxy compound (45 mg, $32 \mu \mathrm{mol})$ and 10\% Pd-C (20 $\mathrm{mg}$ ) in $\mathrm{MeOH}(2 \mathrm{ml})$ was stirred under $\mathrm{H}_{2}$ atmosphere for $12 \mathrm{~h}$, and then filtered through Celite. The filtrate was concentrated in vacuo. To the residue were added pyridine $(1 \mathrm{ml})$ and $\mathrm{Ac}_{2} \mathrm{O}(1$ $\mathrm{ml}$ ) and the mixture was stirred at r.t. overnight. The mixture was diluted with $\mathrm{CH}_{2} \mathrm{Cl}_{2}$ and washed with saturated $\mathrm{NaHCO}_{3}, \mathrm{HCl}(1 \mathrm{~N})$, and $\mathrm{H}_{2} \mathrm{O}$, dried over $\mathrm{Na}_{2} \mathrm{SO}_{4}$ and filtered. The filtrate was concentrated in vacuo and the residue was subjected to flash silica gel column chromatography (1:2 Hexanes: EtOAc) to give $8(40 \mathrm{mg}, 100 \%)$ as a white foam. ${ }^{1} \mathrm{H}$ NMR $\left(\mathrm{CDCl}_{3}, 500 \mathrm{MHz}\right): \delta 6.12\left(\mathrm{~d}, \mathrm{~J}=3.5 \mathrm{~Hz}, 1 \mathrm{H}, \mathrm{H}-1_{\alpha}\right), 6.07(\mathrm{~d}, \mathrm{~J}=9.5 \mathrm{~Hz}, 0.24 \mathrm{H}), 5.67(\mathrm{~d}, \mathrm{~J}=9.0 \mathrm{~Hz}$, 0.23H), 5.43-5.31 (m, 7H), 5.22-5.02 (m, 6H), $4.88\left(\mathrm{~s}, 1 \mathrm{H}, \mathrm{H}-1_{\alpha}{ }^{\prime}\right), 4.84(\mathrm{~s}, 1 \mathrm{H}), 4.73$ (s, 1H, H$1_{\beta}$ '), 4.43-4.11 (m, 13H), 4.05-3.91 (m, 6H), 3.74 (dd. J=4, 10.5Hz, 1H), 3.68-3.67 (m, 1H), 3.64-3.58 (m, $1 \mathrm{H}), 2.24\left(\mathrm{~s}, 3 \mathrm{H}, 1 \times \mathrm{xC}_{3} \mathrm{CO}_{2^{-}}\right), 2.19\left(\mathrm{~s}, 6 \mathrm{H}, 2 \mathrm{xC}_{3} \mathrm{CO}_{2^{-}}\right), 2.18\left(\mathrm{~s}, 3 \mathrm{H}, 1 \mathrm{xC}_{3} \mathrm{CO}_{2^{-}}\right.$ ), $2.17\left(\mathrm{~s}, 3 \mathrm{H}, 1 \times \underline{\mathrm{H}}_{3} \mathrm{CO}_{2^{-}}\right), 2.15\left(\mathrm{~s}, 9 \mathrm{H}, 3 \mathrm{xC}_{3} \mathrm{CO}_{2^{-}}\right), 2.13\left(\mathrm{~s}, 3 \mathrm{H}, 1 \mathrm{xC}_{\underline{3}} \mathrm{CO}_{2^{-}}\right), 2.11(\mathrm{~s}, 3 \mathrm{H}$, $1 \times \underline{H}_{3} \mathrm{CO}_{2^{-}}$, ) $2.10\left(\mathrm{~s}, 3 \mathrm{H}, 1 \times \underline{x}_{3} \mathrm{CO}_{2-}\right), 2.03\left(\mathrm{~s}, 3 \mathrm{H}, 1 \mathrm{xC}_{\underline{3}} \mathrm{CO}_{2^{-}}\right), 2.01\left(\mathrm{~s}, 3 \mathrm{H}, 1 \times \underline{H}_{3} \mathrm{CO}_{2^{-}}\right)$, $1.97\left(\mathrm{~s}, 3 \mathrm{H}, 1 \times \underline{\mathrm{C}}_{3} \mathrm{CO}_{2}-\right)$.

\section{O- $[(\alpha-D-m a n n o p y r a n o s y l)-(1 \rightarrow 6)-(\alpha-D-m a n n o p y r a n o s y l)-(1 \rightarrow 3)-\beta-D-$ mannopyranosyl]- $(1 \rightarrow 4)-(1,2-$ dideoxy- $\alpha$-D-glucopyrano)-[2,1-d]-2-oxazoline (9).}

Compound 8 (22 mg, $18 \mu \mathrm{mol})$ was dissolved in anhydrous $\mathrm{ClCH}_{2} \mathrm{CH}_{2} \mathrm{Cl}(1 \mathrm{ml})$ in an oven-dried flask, under an atmosphere of argon. To the solution were added $\operatorname{TMSBr}(12 \mu 1,91$ $\mu \mathrm{mol}), \mathrm{BF}_{3} \cdot \mathrm{Et}_{2} \mathrm{O}(11 \mu \mathrm{l}, 91 \mu \mathrm{mol})$ and 2, 4, 6-collidine $(12 \mu \mathrm{l}, 91 \mu \mathrm{mol})$. The mixture was stirred at r.t. for $5 \mathrm{~h}$ when TLC indicated the completion of the reaction. The mixture was diluted with 
$\mathrm{CH}_{2} \mathrm{Cl}_{2}$ and washed with saturated $\mathrm{NaHCO}_{3}$ and $\mathrm{NaCl}$ solution. The organic layer was dried over $\mathrm{Na}_{2} \mathrm{SO}_{4}$ and filtered. The filtrate was concentrated in vacuo and the residue was purified by flash silica gel column chromatography $\left(300: 1 \mathrm{EtOAc:} \mathrm{Et}_{3} \mathrm{~N}\right)$ to give the peracetated oxazoline derivative $(17 \mathrm{mg}, 81 \%)$ as a white solid. ${ }^{1} \mathrm{H} \mathrm{NMR}\left(\mathrm{CDCl}_{3}, 500 \mathrm{MHz}\right): \delta 5.92(\mathrm{~d}, \mathrm{~J}=7.5 \mathrm{~Hz}, 1 \mathrm{H}$, $\mathrm{H}-1), 5.60(\mathrm{t}, \mathrm{J}=1.5 \mathrm{~Hz}, 1 \mathrm{H}), 5.41(\mathrm{~d}, \mathrm{~J}=3.5 \mathrm{~Hz}, 1 \mathrm{H}), 5.33-5.24(\mathrm{~m}, 3 \mathrm{H}), 5.19(\mathrm{~d}, \mathrm{~J}=3 \mathrm{~Hz}, 1 \mathrm{H}), 5.17$ $(\mathrm{d}, \mathrm{J}=3 \mathrm{~Hz}, 1 \mathrm{H}), 5.15(\mathrm{~s}, 1 \mathrm{H}), 5.02-5.00(\mathrm{~m}, 2 \mathrm{H}), 4.83(\mathrm{~s}, 1 \mathrm{H}), 4.81(\mathrm{~d}, \mathrm{~J}=1.5 \mathrm{~Hz}, 1 \mathrm{H}, \mathrm{H}-1$ ' $), 4.35$ $(\mathrm{dd}, \mathrm{J}=4,12.5 \mathrm{~Hz}, 1 \mathrm{H}), 4.32-4.05(\mathrm{~m}, 8 \mathrm{H}), 3.93-3.89(\mathrm{~m}, 2 \mathrm{H}), 3.68-3.63(\mathrm{~m}, 2 \mathrm{H}), 3.58(\mathrm{dd}, \mathrm{J}=4$, $11 \mathrm{~Hz}, 1 \mathrm{H}), 3.47-3.43(\mathrm{~m}, 1 \mathrm{H}), 2.23\left(\mathrm{~s}, 3 \mathrm{H}, 1 \times \mathrm{xCH}_{3} \mathrm{CO}_{2}-\right), 2.15\left(\mathrm{~s}, 3 \mathrm{H}, 1 \mathrm{xC}_{3} \mathrm{CO}_{2}-\right), 2.14(\mathrm{~s}, 6 \mathrm{H}$, $\left.2 \mathrm{xC}_{3} \mathrm{CO}_{2^{-}}\right), 2.12\left(\mathrm{~s}, 3 \mathrm{H}, 1 \times \mathrm{xCH}_{3} \mathrm{CO}_{2^{-}}\right), 2.10\left(\mathrm{~s}, 9 \mathrm{H}, 3 \mathrm{xC}_{3} \mathrm{CO}_{2^{-}}\right), 2.05\left(\mathrm{~s}, 6 \mathrm{H}, 2 \mathrm{xC}_{3} \mathrm{CO}_{2^{-}}\right)$, $2.00\left(\mathrm{~s}, 3 \mathrm{H}, 1 \mathrm{xC}_{\underline{3}} \mathrm{CO}_{2^{-}},\right) 1.99\left(\mathrm{~s}, 3 \mathrm{H}, 1 \mathrm{xC}_{\underline{3}} \mathrm{CO}_{2^{-}}\right), 1.96\left(\mathrm{~s}, 3 \mathrm{H}, 1 \mathrm{xC}_{\underline{3}} \mathrm{CO}_{2^{-}}\right)$.

A solution of the peracetylated oxazoline $(25 \mathrm{mg}, 21 \mu \mathrm{mol})$ in $\mathrm{MeOH}(1 \mathrm{ml})$ containing $\mathrm{MeONa}(4.2 \mu \mathrm{mol})$ was stirred at r.t. for $2 \mathrm{~h}$. The $\mathrm{MeOH}$ was evaporated and the residue was dissolved in water and lyophilized to give the oxazoline 9 (15 mg, quantitative) as a white solid. ${ }^{1} \mathrm{H}$ NMR $\left(\mathrm{CDCl}_{3}, 500 \mathrm{MHz}\right): \delta 6.04$ (d, J=7.5Hz, 1H, H-1), 5.04 (s, 1H, H-1' '), 4.89 (s, 1H, H1', '), 4.69 (s, 1H, H-1'), 4.33 (s, 1H, H-2'), 4.14 (s, 1H, H-2','), 4.10 (s, 1H, H-2'’), 4.01 (s, 1H,

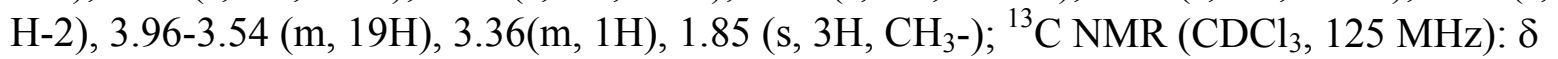
171.1, 102.5, 101.3, 99.9, 99.6, 80.6, 77.8, 74.4, 73.4, 72.7, 70.9, 70.5, 70.3, 70.2, 70.0, 69.9, 69.0, 66.8, 66.7, 65.8, 65.6, 65.1, 61.7, 61.1, 60.9, 12.9; ESI MS: m/z 690.38 (M), 708.3(M+18).

\section{O-(2,3,4,6-tetra-O-acetyl- $\beta$-D-mannopyranosyl)-( $1 \rightarrow 4)$-2-acetamido-1,3,6-tri-O- acetyl-2-deoxy- $\alpha$ - and $\beta$-D-glucopyranose (10).}

A mixture of $4(83 \mathrm{mg}, 84 \mu \mathrm{mol})$ and $\mathrm{NH}_{2} \mathrm{NH}_{2} \cdot \mathrm{H}_{2} \mathrm{O}(3 \mathrm{ml})$ in $\mathrm{EtOH}(27 \mathrm{ml})$ was heated at $90^{\circ} \mathrm{C}$ for $24 \mathrm{~h}$ and the mixture was then concentrated in vacuo. To the oily residue were added pyridine $(2 \mathrm{ml})$ and $\mathrm{Ac}_{2} \mathrm{O}(2 \mathrm{ml})$. The resulting mixture was stirred at r.t. overnight. The reaction mixture was diluted with $\mathrm{CH}_{2} \mathrm{Cl}_{2}$ and washed with $\mathrm{NaHCO}_{3}, 1 \mathrm{~N} \mathrm{HCl}$, and $\mathrm{H}_{2} \mathrm{O}$, dried over $\mathrm{Na}_{2} \mathrm{SO}_{4}$ and filtered. The filtrate was concentrated in vacuo. The crude product was dissolved in $\mathrm{MeOH}(2 \mathrm{ml})$ and $10 \% \mathrm{Pd}-\mathrm{C}(20 \mathrm{mg})$ was added. The mixture was stirred under $\mathrm{H}_{2}$ atmosphere for $12 \mathrm{~h}$ and then filtered through a Celite pad. The filtrate was concentrated in vacuo. The residue was dissolved in pyridine $(1 \mathrm{ml})$ and $\mathrm{Ac}_{2} \mathrm{O}(1 \mathrm{ml})$ and the mixture was stirred at r.t. overnight. The reaction mixture was diluted with $\mathrm{CH}_{2} \mathrm{Cl}_{2}$ and washed with saturated $\mathrm{NaHCO}_{3}$, $1 \mathrm{~N} \mathrm{HCl}$ and $\mathrm{H}_{2} \mathrm{O}$, dried over $\mathrm{Na}_{2} \mathrm{SO}_{4}$ and filtered. The filtrate was concentrated in vacuo and the residue was subjected to flash silica gel column chromatography (1:2 Hexanes: EtOAc) to give 10 (46 mg, 81\%) as a white foam. ${ }^{1} \mathrm{H} \mathrm{NMR}\left(\mathrm{CDCl}_{3}, 500 \mathrm{MHz}\right): \delta 6.11(\mathrm{~d}, \mathrm{~J}=3.0 \mathrm{~Hz}, 1 \mathrm{H}, \mathrm{H}-1)$, 5.71-5.60 (m, 3H), 5.39 (t, J=9.5Hz, 1H, H-4'), 5.22-5.18 (m, 2H), 4.89 (s, 1H, H-1'), 4.45-4.17 (m, 4H), 3.93-3.89 (m, 1H), 3.77-3.75 (m, 2H), 2.18, 2.17, 2.16, 2.12, 2.11, 2.09, 2.00, 1.93 (s each, $3 \mathrm{H}$ each, $1 \times{ }^{1} \underline{H}_{3} \mathrm{CO}_{2}-$ each).

(11).

\section{O-( $\beta$-D-mannopyranosyl)-(1 $\rightarrow 4)-1,2-$ dideoxy- $\alpha$-D-glucopyrano)-[2,1-d]-2-oxazoline}

To a solution of compound $\mathbf{1 0}(60 \mathrm{mg}, 89 \mu \mathrm{mol})$ in anhydrous $\mathrm{ClCH}_{2} \mathrm{CH}_{2} \mathrm{Cl}(1 \mathrm{ml})$ were added TMSBr $(59 \mu \mathrm{l}, 444 \mu \mathrm{mol}), \mathrm{BF}_{3} \cdot \mathrm{Et}_{2} \mathrm{O}(56 \mu \mathrm{l}, 444 \mu \mathrm{mol})$ and 2, 4, 6-collidine $(59 \mu \mathrm{l}, 444$ $\mu \mathrm{mol})$. The mixture was stirred at r.t. for $3 \mathrm{~h}$ when TLC indicated the completion of the reaction. The mixture was diluted with $\mathrm{CH}_{2} \mathrm{Cl}_{2}$ and washed with $\mathrm{NaHCO}_{3}$ and $\mathrm{NaCl}$ solution. The organic layer was dried over $\mathrm{Na}_{2} \mathrm{SO}_{4}$ and filtered. The filtrate was concentrated in vacuo and the residue was subjected to flash silica gel column chromatography $\left(300: 1 \mathrm{EtOAc}: \mathrm{Et}_{3} \mathrm{~N}\right)$ to give the peracetylated oxazoline derivative (44 $\mathrm{mg}, 80 \%)$ as a white solid. ${ }^{1} \mathrm{H} \mathrm{NMR}\left(\mathrm{CDCl}_{3}, 500 \mathrm{MHz}\right): \delta$ 
5.95 (d, J=8Hz, 1H, H-1), 5.59 (dd, J=1.5, 3Hz, 1H, H-3), 5.46 (d, J=3.5Hz, 1H, H-2'), 5.31 (t, $\mathrm{J}=10 \mathrm{~Hz}, 1 \mathrm{H}, \mathrm{H}-4$ '), 5.09 (dd, J=4, 9.5Hz, 1H, H-3'), 4.86 (s, 1H, H-1'), 4.33 (dd, J=5, 12.5Hz, $1 \mathrm{H}, \mathrm{H}-6$ '), 4.24-4.15 (m, 4H), 3.76-3.73 (m, 2H), 3.53-3.50 (m, $1 \mathrm{H}), 2.25\left(\mathrm{~s}, 3 \mathrm{H}, 1 \times \mathrm{xC}_{3} \mathrm{CO}_{2^{-}}\right)$, $2.17\left(\mathrm{~s}, 3 \mathrm{H}, 1 \times \mathrm{xC}_{3} \mathrm{CO}_{2^{-}}\right), 2.15\left(\mathrm{~s}, 6 \mathrm{H}, 2 \mathrm{xC}_{\underline{3}} \mathrm{CO}_{2^{-}}\right), 2.10\left(\mathrm{~s}, 3 \mathrm{H}, 1 \mathrm{xC}_{3} \mathrm{CO}_{2^{-}}\right), 2.09$ (s, $9 \mathrm{H}$, $\left.3 \mathrm{xCH}_{3} \mathrm{CO}_{2-}\right), 2.04$ (s, $\left.3 \mathrm{H}, 1 \mathrm{xCH}_{3} \mathrm{CO}_{2-}\right) ;{ }^{13} \mathrm{C} \mathrm{NMR}\left(\mathrm{CDCl}_{3}, 125 \mathrm{MHz}\right): \delta 170.7,170.6,170.5$, 170.0, 169.6, 169.3, 166.3, 99.3, 99.0, 76.1, 72.5, 70.8, 70.4, 68.4, 67.8, 66.0, 64.8, 63.4, 62.5, $60.4,14.2$.

A solution of the peracetylated oxazoline derivative (44 mg, $71 \mu \mathrm{mol})$ in $\mathrm{MeOH}$ containing $\mathrm{MeONa}(10 \mu \mathrm{mol})$ was stirred at r.t. for $2 \mathrm{~h}$. Then the mixture was concentrated in vacuo. The residue was dissolved in water and lyophilized to give the oxazoline 11 (26 mg, quantitative) as a white solid. ${ }^{1} \mathrm{H} \mathrm{NMR}\left(\mathrm{CDCl}_{3}, 500 \mathrm{MHz}\right): \delta 6.03(\mathrm{~d}, \mathrm{~J}=7.0 \mathrm{~Hz}, 1 \mathrm{H}, \mathrm{H}-1), 4.66$ (s, 1H, H-1'), 4.32 (s, 1H, H-2'), 4.13 (s, 1H, H-2), 3.91-3.88 (m, 4H), 3.72-3.50 (m, 12H), 3.55$3.32(\mathrm{~m}, 3 \mathrm{H}), 1.85\left(\mathrm{~s}, 3 \mathrm{H}, \mathrm{CH}_{3}-\right) ;{ }^{13} \mathrm{C} \mathrm{NMR}\left(\mathrm{CDCl}_{3}, 125 \mathrm{MHz}\right): \delta 168.6,101.2,99.9,77.4,76.4$, $72.8,70.9,70.4,69.3,66.8,65.2,61.7,61.1,13.0$.

\section{Glycopeptide synthesis: A typical procedure for ENGase-catalyzed transglycosylation}

A mixture of the disaccharide oxazoline $11(5.5 \mathrm{mg}, 15 \mu \mathrm{mol})$ and the GlcNAc-peptide $12(5.2 \mathrm{mg}, 5 \mu \mathrm{mol})$ in a phosphate buffer $(50 \mathrm{mM}, \mathrm{pH} 6.0,400 \mu \mathrm{l})$ was incubated at $23^{\circ} \mathrm{C}$ with the enzyme Endo-A or Endo-M (100 milli-units). The reaction was monitored by analytical HPLC on a Waters Nova-Pak C18 column $(3.9 \times 150 \mathrm{~mm})$ at $40^{\circ} \mathrm{C}$ with a linear gradient $(0-90 \%$ $\mathrm{MeCN}$ containing $0.1 \%$ TFA in $25 \mathrm{~min}$, flow rate $1 \mathrm{ml} / \mathrm{min}$ ). Within $2 \mathrm{~h}$, over $90 \%$ of the GlcNAc-peptide was converted to a new species that was eluted slightly earlier than the starting material 12. The enzymatic reaction was stopped by heating in a boiling water bath for 3 min. The product was purified by preparative HPLC on a Waters preparative column (Symmetry 300, $19 \times 300 \mathrm{~mm})$ to afford the glycopeptide $13(5.8 \mathrm{mg}, 82 \%$ from Endo-A reaction, and $5.5 \mathrm{mg}$, $78 \%$ from Endo-M reaction). ESI-MS of 13: Calculated molecular weight, $M=1412.91$; Found, $1413.48(\mathrm{M}+\mathrm{H})^{+}$, and $707.34(\mathrm{M}+2 \mathrm{H})^{2+}$. Detailed characterization of the glycopeptide 13, particularly the assignment of the regio-and stereo-chemistry of the newly formed glycosidic linkage, was achieved by a series of 2D NMR (TOCSY and NOESY) analysis (see Figure S1, Figure S2, and Table S1 for details).

The Endo-A catalyzed transglycosylation between the di- or tetra-saccharide oxazolines and the large peptide GlcNAc-C34 was performed in the same way and the products $\mathbf{1 4}$ and $\mathbf{1 5}$ were purified by HPLC.

Glycopeptide ManGlcNAc 2 -C34 (14): 73\%. ESI-MS: Calculated molecular weight, $\mathrm{M}=$ 4857.33; Found, $1619.81(\mathrm{M}+3 \mathrm{H})^{3+}, 1215.05(\mathrm{M}+4 \mathrm{H})^{4+}$, and $972.20(\mathrm{M}+5 \mathrm{H})^{5+}$.

Glycopeptide $\mathrm{Man}_{3} \mathrm{GlcNAc}_{2}$-C34 (15): 75\%. ESI-MS: Calculated molecular weight, $\mathrm{M}=$ 5181.61; Found, $1727.98(\mathrm{M}+3 \mathrm{H})^{3+}, 1296.22(\mathrm{M}+4 \mathrm{H})^{4+}$, and $\left.1037.20(\mathrm{M}+5 \mathrm{H})^{5+}\right]$. Further structural characterization of glycopeptide 15 was performed by pronase digestion and product analysis. Briefly, glycopeptide 15 was digested with pronase (Sigma) in a phosphate buffer (pH 8.2 ) at $37^{\circ} \mathrm{C}$ for $5 \mathrm{~h}$. The reaction mixture was subject to Sephadex G-10 gel filtration and the carbohydrate positive fractions (detected by anthrone assay) were pooled and subject to ESI-MS and Dionex HPAEC analysis. ESI-MS: Calcd for $\mathrm{Man}_{3} \mathrm{GlcNAc}_{2} \mathrm{Asn}, \mathrm{M}=1024.37$; Found, 
1025.55 $[\mathrm{M}+\mathrm{H}]^{+}$. Under the Dionex HPAEC condition (described above), the product showed a single peak at 14.13 min, which is identical to the standard $\mathrm{Man}_{3} \mathrm{GlcNAc}_{2} \mathrm{Asn}$, the core Asnlinked pentasaccharide. These results confirm that the transglycosylation added the tetrasaccharide to the Asn-linked GlcNAc residue in the peptide to form the core pentasaccharide structure in the resulting glycopeptide. 
Figure S1. The ${ }^{1}$ H NMR Spectra of GlcNAc heptapeptide 12 (panel A) and Glycopeptide 13 (panel B). The spectra were recorded on a Bruker DRX $500 \mathrm{MHz}$ advanced spectrometer equipped with a Bruker TXI cryoprobe. Samples were dissolved in and repeatedly lyophilized from $\mathrm{D}_{2} \mathrm{O}$.
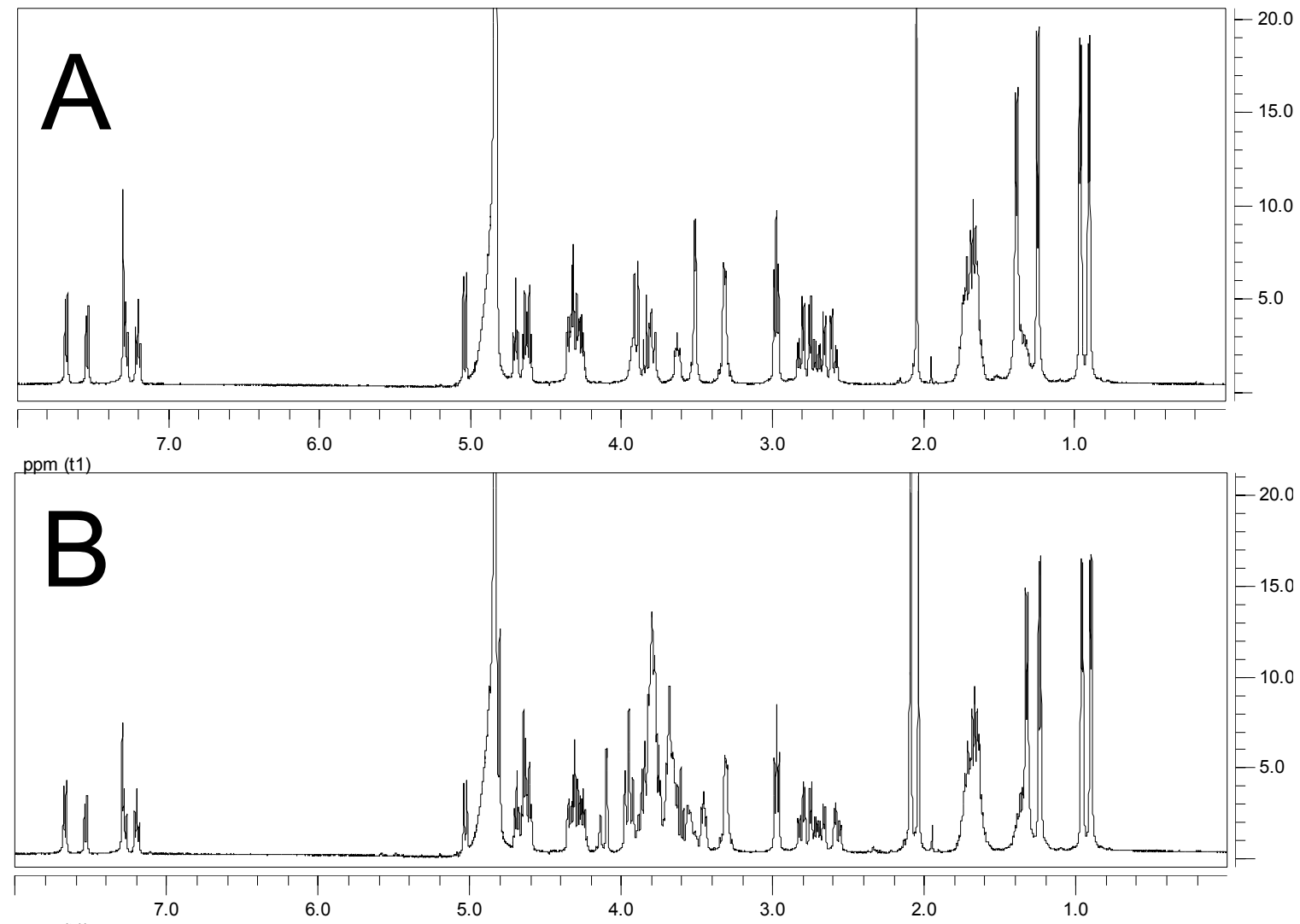

ppm (t1) 
Table S1. ${ }^{1}$ H Assignments (ppm) of glycopeptide 13. Assignments for the peptide portion and the oligosaccharide portion of $\mathbf{1 3}$ were derived from DQF COSY, TOCSY and HSQC spectra. * denotes the Asn residue linked to the GlcNAc. N.d. denotes not determined.

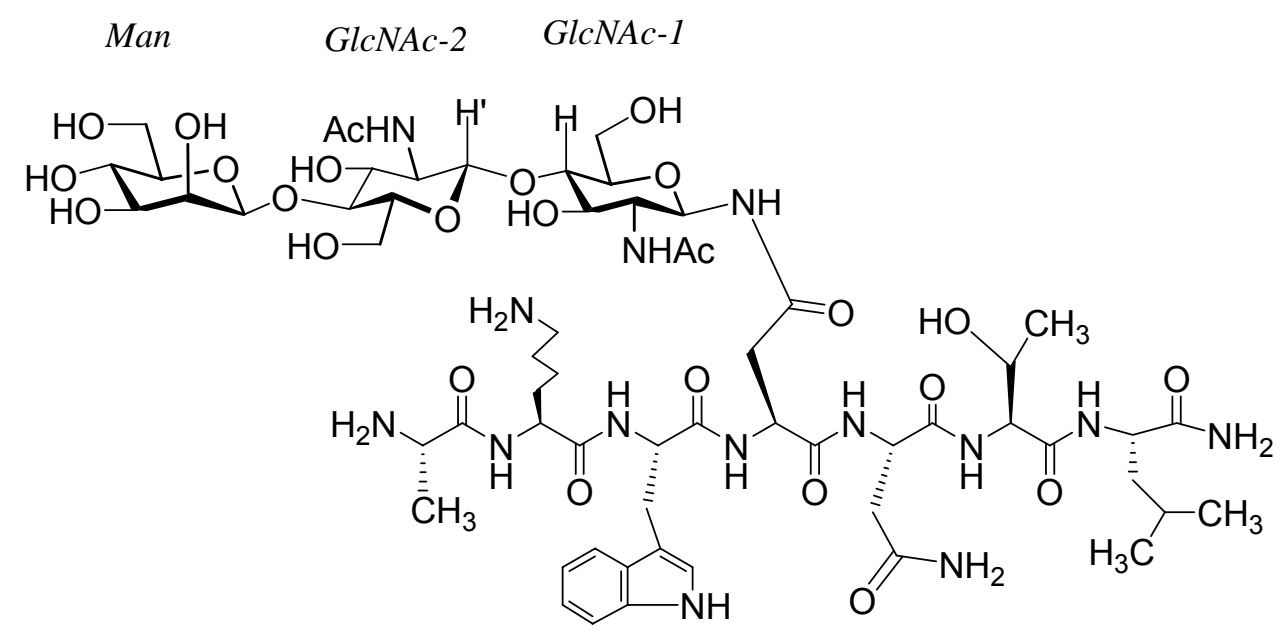

\begin{tabular}{|l|l|l|l|}
\hline & $\mathrm{H} \alpha$ & $\mathrm{H} \beta$ & Other H \\
\hline Ala1 & 3.82 & 1.32 & \\
\hline Lys2 & 4.25 & 1.72 & $\mathrm{H} \gamma 1.35, \mathrm{H} \delta 1.69, \mathrm{H \varepsilon} 2.97$ \\
\hline Trp3 & 4.61 & $2.73,2.81$ & $\begin{array}{l}\text { aromatic } \mathrm{H}, 7.29,7.67,7.20,7.28, \\
\text { and 7.54 }\end{array}$ \\
\hline Asn4* & 4.70 & $3.29,3.32$ & \\
\hline Asn5 & 4.63 & $2.68,2.57$ & \\
\hline Thr6 & 4.34 & 4.27 & $\gamma \mathrm{CH}_{3} 1.24$ \\
\hline Val7 & 4.35 & 1.63 & $\gamma \mathrm{CH}_{3} 0.91$ and 0.96 \\
\hline & $\mathrm{H} 1, \mathrm{H} 2, \mathrm{H} 3, \mathrm{H} 4, \mathrm{H} 5, \quad \mathrm{H} 6, \quad \mathrm{H} 6, \quad$ Ac \\
\hline GlcNAc-1 & $5.03,3.87,3.77,3.68,3.55,3.69,3.83,2.04$ \\
\hline GlcNAc-2 & $4.65,3.82,3.79,3.70,3.65$, n.d., 3.84, 2.09 \\
\hline Man & $4.80,4.09,3.69,3.61,3.46,3.77,3.98$ \\
\hline
\end{tabular}


Figure S2. TOCSY (panel A) and NOESY (panel B) of glycopeptide 13. The relatively large coupling constant for the $\mathrm{H}-1^{\prime}\left(J_{1}, 2,=8.5 \mathrm{~Hz}\right)$ of the second GlcNAc indicated that the newly formed glycosidic bond was in $\beta$-configuration. The $\beta-1,4-$ linkage type of the newly formed glycosidic bond was determined by the apparent NOE correlation between H-1' and H-4 in the NOESY analysis (pabel B).
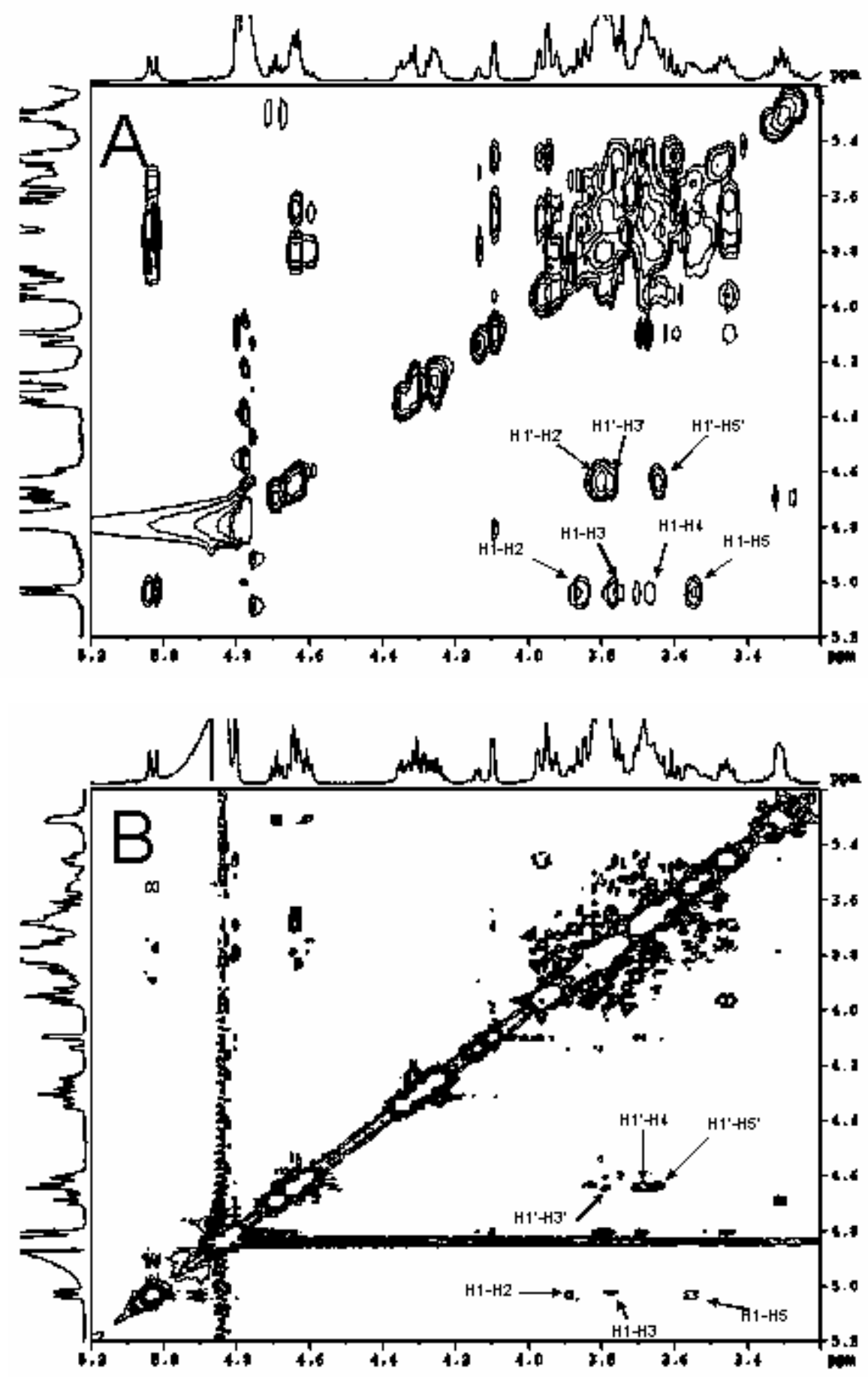
Figure S3. The ESI-MS spectrum of glycopeptide 13

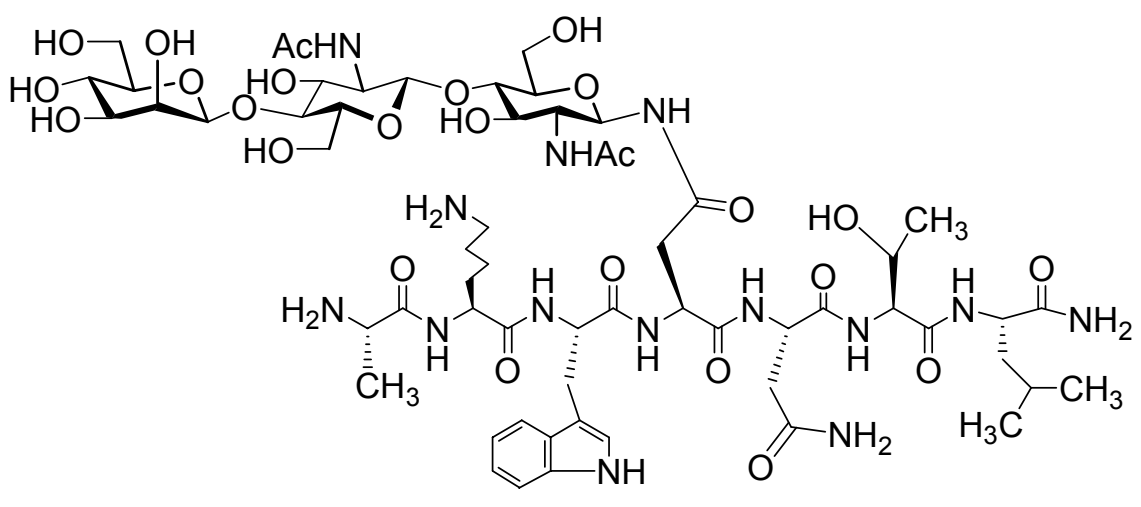

13

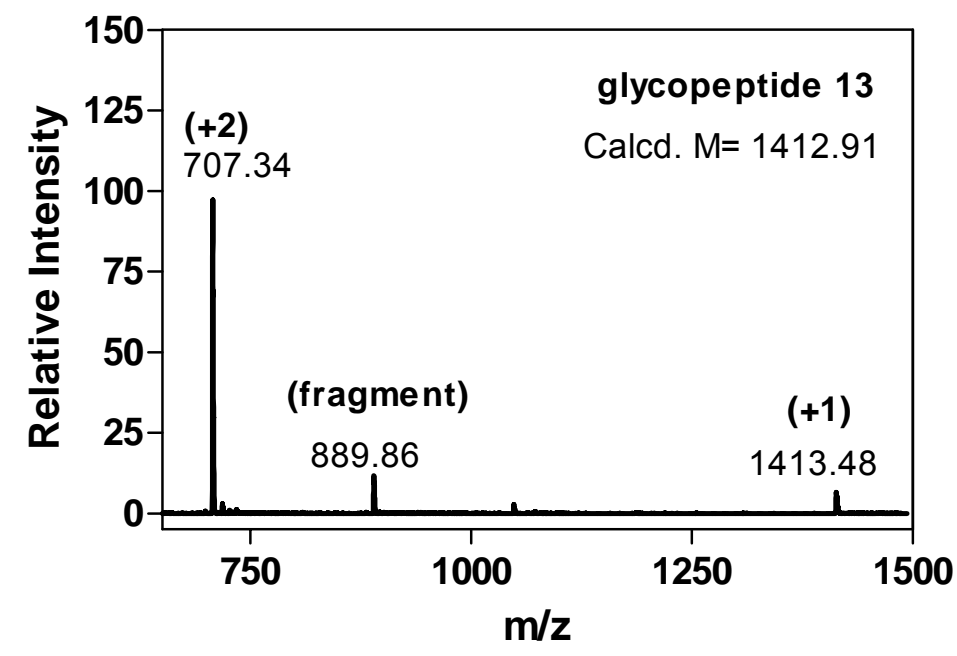


Figure S4. The ESI-MS spectrum of glycopeptide 14

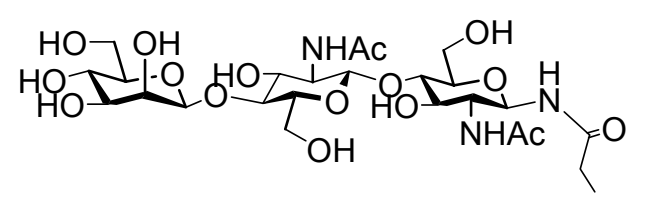

Ac-W-M-E-W-D-R-E-I-N-N-Y-T-S-L-I-H-S-L-I-E-E-S-Q-N-Q-Q-E-K-N-E-Q-E-L-L-NH ${ }_{2}$

14

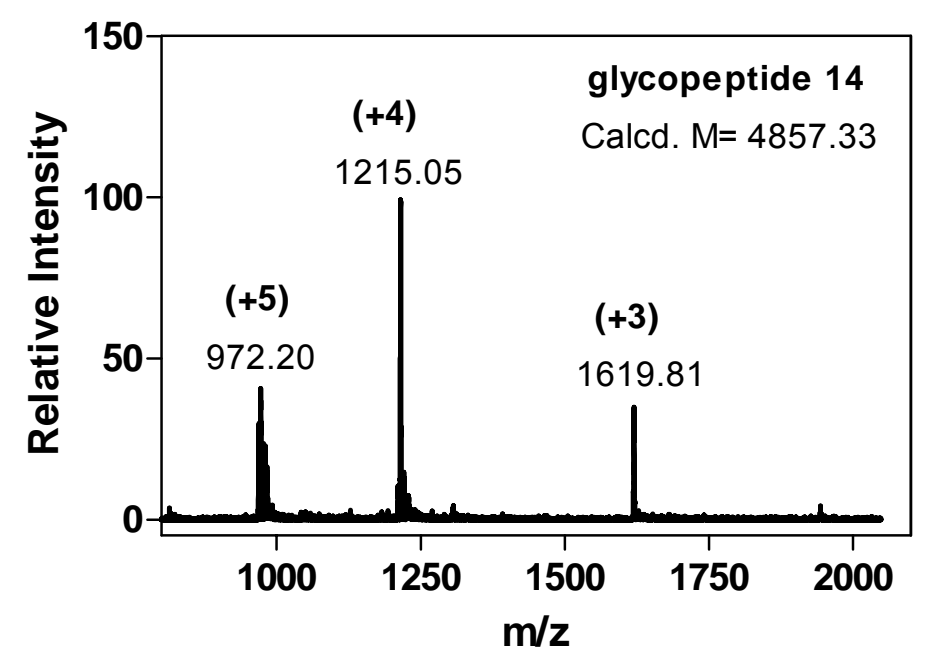


Figure S5. The ESI-MS spectrum of glycopeptide 15
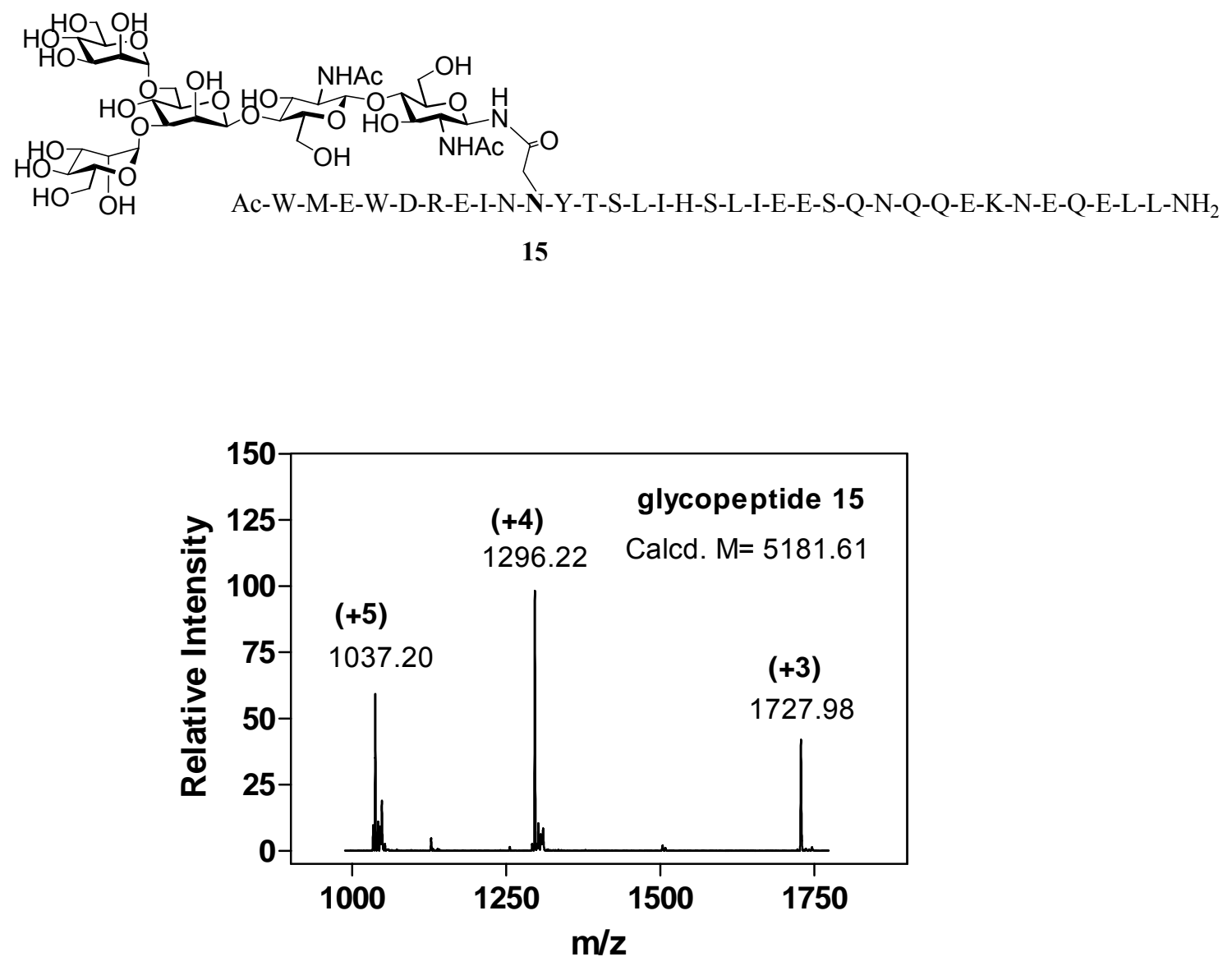